УДК 697.311

\title{
Взаимодействие систем теплоснабжения для устранения потерь через наружную поверхность многослойной ограждающей конструкции
}

\author{
Н. А. Прусенков \\ Одесский национальный политехнический университет, пр. Шевченко,1, г. Одесса, 65044, Украина
}

\begin{abstract}
При эксплуатацчии многослойной ограждающей конструкции (МОК) постоянно возникает превыщение расчетной температуры наружной поверхности наружного слоя, сравнительно с температурой пространства, расположенного снаружи этой ограды. На наружной поверхности ограждения, соответственно, формируется выброс тепла, возникающий из-за разницы нормативного и фактического значений температур. Исключение эксплуатационных потерь тепла является ичелью публикации. Вариантом достижения иели представляется создание альтернативной системы теплоснабжения, следящей за изменениями температур в наружном пространстве МОК и компенсирующей или исключающей соответствующие выбросы подачей энергии. Перенос и компенсационные поступления ее выполнимы при устройстве дополнительного слоя МОК с подвижной средой и поверхностями, соприкасающимися с сущзествующими. Теоретическая база расчета затрат энергии требует определения потерь в подвижном слое на перемещзение подвижной среды, переносящей тепло. Включение в МОК подвижного слоя помимо реализации принципов тектологии требует пересмотра допустимости дистрибуции в подвижном слое, заменившем замкнутую вентиляционную прослойку, узаконенную существуюциими нормами и методиками определения потерь фасадными системами. Схема взаимодействия теплотранспортных систем, в отличие от прототипа из замкнутых слоев, допускает изменение тепловосприятия и теплоотдачи в подвижном слое. Взаимодействие существующей и регулирующей систем в подвижной среде МОК прогнозирует модернизацию регулирования потерь энергии в период эксплуатации.
\end{abstract}

Ключевые слова: Выброс энергии; Подача тепла; Перемещение среды; Подвижный и замкнутый слои; Температура поверхности; Теплоотдача; Тепловосприятие; Термическое сопротивление

\section{Взаємодія систем теплопостачання для усунення втрат через зовніш- ню поверхню багатошарової огороджувальної конструкції}

\section{М. О. Прусенков}

Одеський національний політехнічний університет, пр. Шевченка, 1, м. Одеса, 65044, Україна

\begin{abstract}
При експлуатації багатошарових огороджувальних конструкцій (БОК) постійно виникає перевищення розрахункової температури зовнішньої поверхні зовнішнього шару порівняно з температурою простору, розташованого зовні огорожі. На зовнішній поверхні огорожі постійно формується викид тепла, щчо виникає через різницю нормативного та фактичного значень температур. Метою публікації є усунення експлуатаційних втрат. Варіантом ії досягнення є створення альтернативної системи теплопостачання, яка стежить за змінами температур у зовнішньому просторі БОК та компенсує або уникає відповідних викидів наданням енергії. Перенесення і компенсаційні надходження тепла забезпечують влаштуванням додаткового шару з рухомим середовищем та поверхнями, дотичними до існуючих замкнених. Теоретична база розрахунку витрат енергіi вимагає визначення втрат у рухомому шарі на переміщення рухомого середовища, шо транспортує тепло. Включення до БОК рухомого шару крім реалізації принципів тектології вимагає перегляду припущення дистрибуції у рухомому шарі, щуо замінює замкнений вентиляційний прошарок, узаконений методиками визначення втрат тепла фасадними системами. Схема взаємодії тепло-транспортних систем, на відміну від прототипу з замкнутих шарів, дозволяє унеможливити сприйняття тепла і тепловіддачі поверхнями рухомого шару. Забезпечення взаємодії існуючої і регулюючої систем переходу енергї та теплообміну прогнозує модернізацію регулювання втрат при експлуатації.
\end{abstract}

Ключові слова: Викид енергї̈; Переміщення середовища; Рухомий і замкнутий иари; Температура поверхні; Тепловіддача; Теплосприйняття; Термічний опір

(C) The Author(s) 2017. This article is an open access publication

This work is licensed under the Creative Commons Attribution 4.0 International License (CC BY) http://creativecommons.org/licenses/by/4.0/ 


\section{1 Введение}

Действующая ДБН [1], декларируя постоянство термических сопротивлений замкнутых слоев многослойной ограды $\left(R_{\mathrm{x} 3}=\delta_{\mathrm{x} 3} / \lambda_{\mathrm{x} 3}=\right.$ const, $\left.\mathrm{m}^{2} \mathrm{~K} / \mathrm{BT}\right)$ [2], ограничивается регламентированием перепада температур $\left(\Delta t=t_{\mathrm{B}}-t_{\mathrm{H}}, \mathrm{K}\right)$, обеспечивая создание заданной на внутренней поверхности температуры $t_{\mathrm{B}}=$ const, при любой фактической температуре в пространстве снаружи $\left(t_{\mathrm{H}}=t_{\mathrm{H}}\right)$. В круглогодичном цикле эксплуатации формируется уменьшение перепада фактических температур $\left(\Delta t_{\phi}=t_{\mathrm{B}}-t_{\mathrm{H \phi}}<\Delta t\right)$, постоянно стимулирующего нерегулируемый выброс тепловой энергии через наружную поверхность. Апеллирование современных норм к экстремальным температурам, при постоянстве их перепадов в слоях $\left(\Delta t=\sum \Delta t_{\mathrm{x}}=\mathrm{const}\right)$ и направления потока энергии в системе, создает фактические выбросы $\left(Q_{\text {выб.нф }}=\left(\Delta t-\Delta t_{\phi}\right) \cdot F \cdot t_{\text {сек }} / \mathrm{R}_{\text {мок }}\right.$, Дж) через наружную поверхность при постоянном теплопоступлении от единственного источника $\left(Q_{\text {cuc.1 }}=\right.$ const $)$. Значит, узаконенная ДБН [1] система перехода потоком тепла замкнутой МОК не предусматривает регулирование в период эксплуатации фактического температурного перепада, возникающего с повышением температуры пространства снаружи во время эксплуатации [1-3]. Адекватное уменьшение выброса тепла через наружную поверхность МОК в период эксплуатации, вплоть до его обнуления $\left(Q_{\text {выб.нф }}=0\right)$, снижает затраты источ-

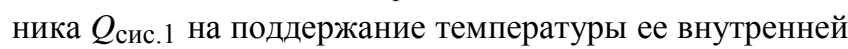
поверхности.

Согласно законам термодинамики [1-5] переходы тепла между соприкасающимися поверхностями, в том числе и в окружающем МОК пространстве, исключаются уравниванием их температур.

Система подачи тепла, созданная согласно ДБН [1], формирует переход потока тепла через замкнутую МОК, при заданном перепаде $\Delta t=$ const:

$$
\frac{Q_{c u c .1}}{F} t_{\text {сек }}=\frac{N_{\text {сис. } 1}}{F}=-\lambda_{\text {мок }} \operatorname{grad} t=q_{\text {сис. } 1}=\frac{\Delta t}{R_{\text {мок }}},
$$

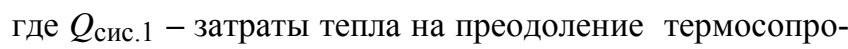
тивления МОК, состоящей из замкнутых слоев, Дж;

$F=l \cdot h-$ площадь поверхности МОК, м $^{2}$;

$l$ - расчетная длина каналов (ширина) МОК, м;

$h$ - высота канала МОК, м;

$t_{\text {сек }}$ - продолжительность поступления энергии, сек;

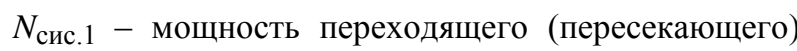

МОК потока тепла, созданного системой $1, \mathrm{BT}$;

$q_{\text {сис.1 }}$ - плотность теплового потока, пересекающего внутреннюю поверхность МОК, в системе $1, \mathrm{BT} / \mathrm{M}^{2}$;

$\Delta t$ - перепад температур на поверхностях МОК, К;

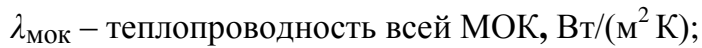

$\lambda_{\mathrm{x}}$ - теплопроводность данного слоя (х), Вт/( $\left.{ }^{2} \mathrm{~K}\right)$;

$\operatorname{grad} t-$ градиент температур потока через замкнутую МОК.

Для замкнутой МОК [1-5] при независимой температуре наружного пространства $t_{\mathrm{H}}$ это достижимо только при условии соответствующего изменения температуры на внутренней поверхности $t_{\mathrm{B}}$. При единственно- сти системы (сис.1), созданной согласно ДБН [1], в затратах источника $Q_{\text {сис.1 }}$ в замкнутой МОК учтены потери на преодоление фактического термического сопротивления и выброс избытка тепла, проходящего через наружную поверхность $\left(Q_{\text {сис.1 }}=Q_{\text {сис.1ф }}+Q_{\text {выб.н }}\right)$. Указания ДБН [1] нормируя температуры поверхностей МОК определением выброса пренебрегают, ограничиваясь регламентацией затрат, обеспечивающих ее необходимое термическое сопротивление.

Достижением 20-го века в теории расчета потерь тепловым потоком, пересекающим изоляционную конструкцию, считают внедрение многослойных ограждающих конструкций (МОК) [1,5]. Их выполняют из неподвижных сред, формируя замкнутую МОК. Это требует равенства температур соприкасающихся поверхностей, позволяя дистрибутировать потери энергии замкнутыми слоями прямо пропорционально перепадам температур и обратно пропорционально удельным термическим сопротивлениям (рисунок 1). При этом перепады температур поверхностей всей МОК и соответствующих слоев (внутренний - вс, подвижный - пс, наружный - нс), К:

$$
\Delta t=\Delta t_{\mathrm{Bc}}+\Delta t_{\Pi \mathrm{C}}+\Delta t_{\mathrm{Hc}}
$$

суммарное удельное термическое сопротивление слоев

$$
R_{\text {мок }}=R_{\mathrm{Bc}}+R_{\text {пс }}+R_{\mathrm{Hc}}
$$

Целью публикации является рассмотрение предпосылок исключения выброса потерь тепла через наружную поверхность МОК в окружающее пространство при ее эксплуатации $\left(Q_{\text {выб.н }}=0\right)$.

\section{2 Включение подвижного слоя МОК}

При рассмотрении переноса теплоносителя в подвижных средах обычно ограничиваются оценкой потерь давления на преодоление сопротивления его движению в сети $\left(\Delta p_{\text {сис. } 2}\right.$, Па) $[2,4-7]$ :

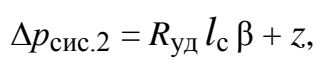

где $R_{\text {уд }}$, Па/м - удельное сопротивление давления в сети (удельная потеря давления);

$l_{\mathrm{c}}$ - длина участка сети (трубопровода), м;

$\beta$ - коэффициент шероховатости;

$z=\left(\sum \xi V_{\text {пс }}^{2} \rho\right) / 2$ - потеря давления в местных сопротивлениях сети, Па;

$\sum \xi$ - сумма коэффициентов местных сопротивлений участка сети;

$V_{\text {пс }}$ - скорость теплоносителя в подвижном слое, $\mathrm{M} /$ сек;

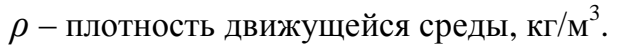

Примечание: при большом числе способов перемещения теплоносителя $\left(A_{\text {сис. } 2}=f(\Delta p)\right)$ их осуществление в публикации не рассматривается, как уже решенная известными источниками задача [4-9].

Потери тепла подвижными теплоносителями при расчете динамических характеристик сети обычно не учитывают из-за незначительности затрат источника 
движения на теплообмен и теплоперенос. Температуры поверхностей соприкасающихся слоев МОК при этом регулируются изменением свойств и параметров поступающей среды теплоносителя. Для замкнутой воздушной прослойки ДБН [1] ограничивается учетом постоянства термического сопротивления подвижного слоя $\left(R_{\mathrm{B \Pi}}=R_{\text {пс }}\right.$, см. с. 23 [1] Табл. И.1). Тепловосприятие и теплоотдача воздушной прослойки $\left(\alpha_{\text {впс }}\right.$, и $\left.\alpha_{\text {нпс }}\right)$, аналогично контактам поверхностей МОК с окружающим пространством, учитываться при теплообмене в подвижной среде не должны, что оставлено без внимания действующими нормами и литературными источниками [1-5].

Оценивания температур поверхностей подвижного слоя норматив не предусматривает, пренебрегая использованием свойств дистрибуции и суперпозиционирования при перемещении теплоносителя. Известными «Рекомендациями» [7] приближенно учитывается сопротивление переходу тепла через воздушную прослойку, влияющее на перепад между температурами поверхностей подвижного слоя. Документы [1-7] исключают учет фактического теплосодержания в среде подвижного слоя, взаимодействующего с потоком, пересекающим МОК. При использовании в составе МОК слоя с подвижной средой, справедливость указанных утверждений [1-7] должна быть пересмотрена.

Использование подвижного слоя требует включения в расчеты дополнительной системы, регулирующей подачу тепла и энергии в период эксплуатации $Q_{\mathrm{cuc.2}}$, компенсирующих потери на перемещение подвижной среды источником этой системы $A_{\text {сис.2н }}=f(\Delta p)$ (см. Примечание к ф.2.). Это осуществляется регулированием температур на поверхностях подвижного слоя и скорости теплоносителя в период эксплуатации [4-9]:

$$
\begin{aligned}
& Q_{\text {сис } 2}=C_{v} \Delta T_{\text {пс }} w_{\text {пс }}=\mathrm{C}_{v} \Delta T_{\text {пс }} L_{\text {пс }} t_{\text {сек }}= \\
& =C_{v} \Delta T_{\text {пс }} V_{\text {пс }} f_{\text {пс }} t_{\text {сек }} \text {, }
\end{aligned}
$$

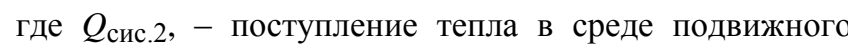
слоя (при эксплуатации постоянно меняется $Q_{\text {сис.2ф), }}$ Дж;

$C_{v},-$ удельная теплоемкость объема среды подвижного слоя, Дж/( $\left.\mathrm{M}^{3} \mathrm{~K}\right)$;

$\Delta T_{\text {пс }}-$ разность температур среды, поступающей в теплоноситель $\left(t_{\text {пс }}\right)$, и в пространстве снаружи МОК $\left(t_{\mathrm{H \phi}}\right), \Delta T_{\text {пс }}=t_{\text {пв }}-t_{\mathrm{H \phi}}, \mathrm{K}$;

$w_{\text {пс }}=\delta_{\text {пс }} l h=f_{\text {пс }} h-$ объем теплоносителя в подвижном слое, м $^{3}$;

$L=V_{\text {пс }} f_{\text {пс }}-$ расход теплоносителя среды, ${ }^{3} /$ сек;

$t_{\text {сек }}$ - продолжительность пребывания среды в подвижном слое, сек;

$V_{\text {пс }}-$ скорость подвижной среды в ее слое, м/сек;

$f_{\text {пс }}=\delta_{\text {пс }} l, \mathrm{м}^{2}-$ площадь поперечного сечения подвижного слоя;

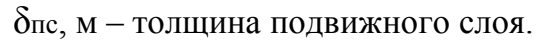

Во избежание возникновения противотока тепла в замкнутом внутреннем слое (вс) МОК необходимо обеспечить равенство температур соприкасающихся наружной $t_{\text {вп }}$ и внутренней $t_{\text {пв }}$ поверхностей внутреннего и подвижного слоев.

Схема, представленная на рисунке 1 , иллюстрирует взаимодействие слоев МОК, обеспечивающих послойный переход теплового потока, с учетом теплоотдачи и тепловосприятия поверхностей каждого из замкнутых (вс и нс) слоев, при теплообмене в подвижном слое (пс), расположенном между ними. Она отличается от аналога учетом регулирования теплоотдачи поверхностей подвижного слоя при фактическом взаимодействии поступлений $\left(\sum Q_{\text {сис.ф }}=Q_{\text {сис. } 1}+Q_{\text {сис.2ф }}\right)[10]$. Это обосновывает исключение потерь тепловосприятия и теплоотдачи в подвижном слое, так же как и влияние на него поступлений через соприкасающиеся с ним поверхности замкнутых слоев. В примере, приведенном на рисунке 1, рассмотрено взаимодействие систем в направлении между слоями МОК при уравнивании температур соприкасающихся поверхностей $\left(t_{\text {пв }}=t_{\mathrm{Bп}}\right.$ и $\left.t_{\mathrm{\Pi H}}=t_{\mathrm{H \Pi}}\right)$.

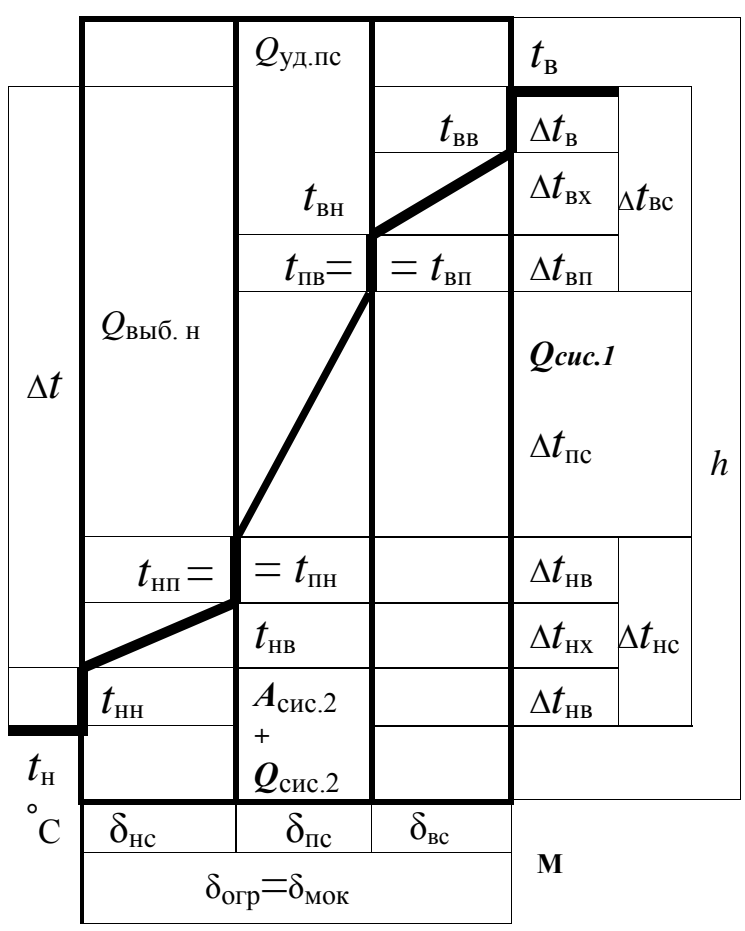

Обозначения:

1. Перепад температур на поверхностях МОК $(\Delta t)$ определяется указаниями ДБН [1] для экстремальных условий эксплуатации.

2. На схеме индексы обозначают:

А) Первая буква индекса толщины слоя:

- в - внутренняя поверхность МОК;

- П - поверхность подвижного слоя;

- н - наружная поверхность МОК.

Б) Вторая буква индекса - наименование соприкасающегося с данным слоя.

В) $\Delta t-$ перепад температур на поверхностях.

3. $\delta_{\text {огр }}=\delta_{\text {но }}+\delta_{\text {пс }}+\delta_{\text {мсг }}-$ толщина ограждения или МОК и слоев.

Рисунок 1 - Изменения температур при взаимодействии потоков в замкнутых и подвижном слоях МОК 
Пересмотром порядка позиционирования операций в подвижном слое МОК при взаимодействии пересекающихся потоков, с учетом потенциала регулирования теплосодержания и скорости теплоносителя источником тепла, принадлежащим системе 2, формирующей

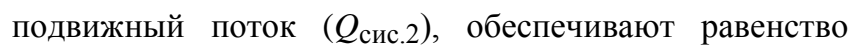
температур на соприкасающихся поверхностях подвижного (пс) и наружного (нс) слоев.

Уравнивание температур наружного пространства и наружной поверхности наружного слоя $t_{\mathrm{H}}$ в любой момент эксплуатации достигается изменением поступле-

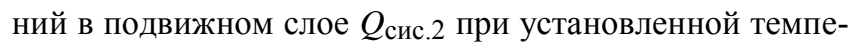
ратуре внутренней поверхности подвижного слоя, в зависимости от температуры снаружи МОК. При этом изменением теплопоступлений в подвижном слое $Q_{\text {сис.2 }}$ и кинетической энергии его среды $A_{\text {сис.2 }}=f(\Delta p)$ добиваются исключения выброса через наружную поверх-

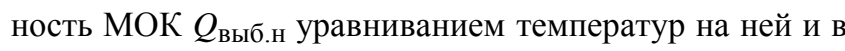
пространстве снаружи $t_{\mathrm{H}}$, что является целью данной публикации.

На рисунке 1 показан частный случай предотвращения потерь тепла наружной поверхностью внутреннего замкнутого слоя МОК (вс) подачей теплоносителя с рассчитываемой температурой $\left(t_{\text {пв }}=t_{\mathrm{B \Pi}}\right)$.

При внедрении подвижного слоя в состав МОК следует уточнить рекомендации и указания или предложить решения, учитываемые конструктивно и технологически в период эксплуатации, обеспечивающие:

a) температуру конденсации влаги на наружной поверхности подвижного слоя $t_{\text {пн; }}$;

б) расчет и устанавливание средней температуры теплоносителя в удаляемой среде: $\Delta T_{\text {уд.ср }}=\left(t_{\text {пв }}+t_{\text {пн }}\right) / 2$;

в) адекватность изменения скорости теплоносителя и температуры снаружи МОК...

Проблемы утилизации удаляемого в подвижной

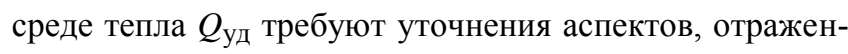
ных в данной публикации, и учета индивидуальных особенностей каждой из взаимодействующих систем. Например, в специальных дополнениях к нормам (или разделах проектов), целесообразно учесть:

- условия формирования противотока тепла через внутренний слой для модернизации отопления;

- конденсацию влаги поверхностями подвижного слоя;

- утилизацию тепла в среде подвижного слоя;

- мероприятия и принципы минимизации потерь, созданные суммарной системой (сис. 3), сформированной взаимодействием составляющих (сис. 1 и сис. 2).

\section{3 Задачи, решаемые включением в МОК подвижного слоя}

Включение в состав МОК подвижного слоя (сис.2) прогнозирует создание объединенной системы (сис.3), обеспечивающей взаимодействие замкнутых и подвижного слоев, что позволяет решить дополнительно ряд задач, непосильных для замкнутых МОК:

1. Регулирование температур на поверхностях подвижного слоя $\left(t_{\mathrm{Bп}}\right.$ и $\left.t_{\text {пн }}\right) \mathrm{MOK}$, соприкасающихся с за- мкнутыми слоями (вс и нс), изменением теплопоступлений в подвижной среде [1-10].

2. Компенсационный теплообмен между пересекающимися в подвижном слое потоками тепла, взаимодействующих систем $Q_{\text {сис.1 и }} Q_{\text {сис.2, исключающий за- }}$ траты, на объединение и взаимодействие систем, формируя систему более высокого уровня.

3. Обеспечение постоянства температуры внутренней поверхности МОК $t_{\mathrm{B}}=$ const и поступления тепла в замкнутой системе $Q_{\text {сис.1 }}$ согласно принципам расчета перехода тепла в ДБН [1], что корректируется поступлениями тепла и энергии в среде подвижного слоя (сис. 2).

4. Удаление части тепла в среде подвижного слоя $Q_{\text {уд.пс }}$ и преодоление термического сопротивления наружного замкнутого слоя $Q_{\text {выб.н }}$.

На рисунке 1 показан частный случай предотвращения потерь тепла наружной поверхностью внутреннего замкнутого слоя МОК подачей теплоносителя с рассчитываемой температурой $\left(t_{\text {пв }}=t_{\text {вп }}\right)$.

\section{Выводы}

1. Узаконенная ДБН [1] система (сис. 1) перехода тепла через МОК не предусматривает регулирование затрат при повышении наружной температуры в период эксплуатации.

2. Уменьшение выброса через наружную поверх-

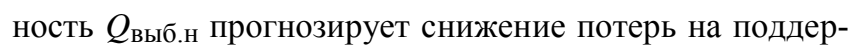
жание температур поверхностей МОК.

3. Допущенное нормами $[1,5]$ упрощение учета коэффициентов тепловосприятия и теплоотдачи $\left(\alpha_{\mathrm{B}}\right.$ и $\left.\alpha_{\mathrm{H}}\right)$ всех слоев требует уточнения для дистрибуции при наличии подвижных сред в МОК.

4. Потери тепла и энергии в подвижной среде при замене перехода теплового потока через замкнутый слой на теплообмен в среде подвижного потока МОК не возникают [1-7].

5. Система теплопоступлений в среде подвижного

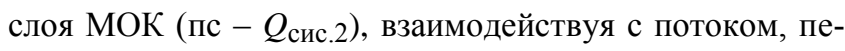
ресекающим ее замкнутые слои $\left(Q_{\text {сис.1 }}\right)$, позволяет регулировать выбросы через поверхность.

6. При включении в МОК слоя с подвижной средой указания ДБН [1] должны пересматриваться и дополняться учетом их свойств и особенностей.

7. Включение подвижного слоя в МОК регламентирует использование системы обеспечения поступлений тепла $Q_{\text {сис.2 }}$ и энергии, компенсирующей потери давления для перемещения подвижной среды $A_{\text {сис.2 }}=f(\Delta p)$, [6-9].

8. Теплообмен между пересекающимися потоками систем $Q_{\text {сис.1 и }} Q_{\text {сис.2 не требует затрат источников на }}$ взаимодействие в подвижном слое. Свойства проявляемые при этом, позволяют регулировать выброс тепла через наружную поверхность МОК $Q_{\text {выб.н и в удаляе- }}$

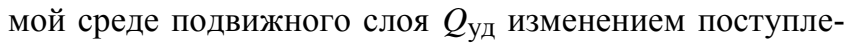

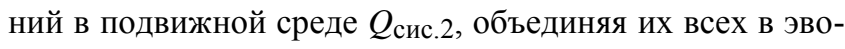
люционную систему (сис.3) более высокого уровня. 


\section{Литература}

1. ДБН В.2.6-31:2006 Тепловая изоляция зданий. - К.: Минстрой Украины, Укрархстройинформ, 2006. - 64 с.

2. Тихомиров К. В. Теплотехника, тепло-газоснабжение и вентиляция. - М: Стройиздат, 1981.-272 с.

3. Прусенков Н.А. Использование принципов тектологии для регулирования потерь тепла потоками. - Одесса: ОНПУ, Пути реализации кредитно-модульной системы, №10, 2015.- С. 58-63.

4. Соколов Е.Я., Бродянский В.М. Энергетические основы трансформации тепла и охлаждения.- М: Энергоиздат, 1981.- 320 с.

5. СНиП ІІ-3-79** Гл. 3 Строительная теплотехника М.: Госстрой СССР, 1986. - 32 с.

6. Егорушкин В.Е., Цеплович Б.И. Основы гидравли ки и теплотехники. - М.: Машиностроение, 1981. $268 \mathrm{c}$.

7. Рекомендации проектирования навесных фасадных систем с вентилируемым воздушным зазором. - М: Москомархитектура, 2002.- 105 с.

8. Прусенков Н.А. Компенсация потерь тепла в «подвижном» слое.- Одесса: ОНАХТ, Холодильная техника и технология, №1(135), 2012. - С. 46-49.

9. Прусенков Н.А. Предпосылки учета особенностей перехода тепловым потоком ограждений с «подвижным» слоем. - Одесса: ОГАСА, Вестник ОГАСА, №50, ч.1, 2013. - С. 251-255.

10. Прусенков Н.А. Схема определения составляющих потерь тепла в подвижном слое МОК. - Одесса: ОНАХТ, Холодильная техника и технология, №5 (151), 2014.- C. 55-60.

Отримана в редакції 22.09.2017, прийнята до друку 08.12.2017

\title{
Interaction of Heat Supply Systems to Eliminate Losses Through the Outer Surface of a Multilayered Enclosure Structure
}

\author{
N. A. Prusenkov \\ Odessa National Polytechnic University, av. Shevchenko, 1, Odessa, 65044, Ukraine
}

\begin{abstract}
In the process of exploitation, constantly arises the excess of the estimated temperature of the outer surface outer layer of the multi-layered enclosing structure (MES), compared with the temperature of the space located outside of this structure. On the external surface of the fence a heat release due to the difference in the normative and actual temperatures is generated. The elimination of operational losses is the purpose of publication. A variant of achieving the goal is the creation of an alternative heat supply system that monitors temperature changes in the outer space of the MES and compensates or eliminates the corresponding outflows by power supply. The transfer and compensating inputs of power are feasible when an additional layer with a moving medium and surfaces that are in contact with the existing ones are being built. The theoretical basis for energy expenditure calculation requires the determination of losses in the moving layer according to the transfer of the mobile medium which transfer heat. Inclusion of the moving layer into the $M E S$, in addition to the implementation of the principles of tectology, requires a review of the admissibility of distribution in the moving layer, that have replaced the closed ventilation layer, legalized by existing standards and methods for determining the losses by facade systems. The scheme of interaction of heat-transport systems, in contrast to the prototype from closed layers, allows the alteration of heat perception and heat transfer in the moving layer. Interaction of the existing and regulatory systems in the MES mobile medium predicts the modernization of the regulation of losses during the period of exploitation.
\end{abstract}

Key words: Energy Release; Heat Supply; Medium Displacement; Moving And Closed Layers; Surface Temperature; Heat Transfer; Heat Perception; Thermal Resistance

\section{References}

1. DBN B. 2.6-31:2006 Teplovaya izolyatsiya zdaniy $i$ sooruzheny. K: Minstroy Ukrainy "Ukrstroyinform", 2006, 45 p., s il. (in Russian)

2. Tikhomirov K.V. (1981) Teplotekhnika, teplogazosnabzhenie $i$ ventiliatsia. M.: Stroyizdat, 272 p. (in Russian)

3. Prusenkov N. A. (2015) Ispolzovaniye printsipov tektologii dlia regulirovaniia poter tepla potokami v MOK

- Odessa: ONPU, Puti realizatsii kreditno-modulnoi siste$m y$, No.10, 58-63 (in Russian)

4. Sokolov E. Y., Brodianskii V. M. (1981). Energeticheskie osnovy transformatsii tepla i protscessov okhlazhdeniia. Moskow: Energoizdat, 320 p. (in Russian)

5. SNiP II-3-79** (1992) Stroitelnay teplotehnika. Moskow: Gosstroi, 75 p. (in Russian)

6. Iegorushkin V. E., Tseplovich B. I. (1981) Osnovy gidravliki i teplotekhniki. Moskow: Mashinostroenie, 268 p. (in Russian)
7. Rekomendatsii po proekirovaniy navesnih fasadnyh sistem s ventiliruymim vozdushnym zazorom dlya stroi-tel'stva $i$ rekonstruktsii zdaniy. (2002). M: Moskomarkhitektura, 104 p. (in Russian)

8. Prusenkov N. A. (2012) Heat loss compensation in «mobile» protection layer. Refrigeration engineering and technology, 1(135), 46-49 (in Russian)

9. Prusenkov N. A. (2013) Predposylki uchota osobenostei perekhoda teplovym potokom ograzhdenii s podvizhnym sloiem. Vesnik OGASA, No.50 (1), 251-255.(in Russian).

10. Prusenkov N. A. (2014) Scheme determining components heat loss in a moving layer of the multilayers walling. Refrigeration engineering and technology, 5(151), 55-60. (in Russian) DOI: http://dx.doi.org/10.15673/0453$\underline{8307.5 / 2014.28705}$

Received 22 September 2017 Approved 08 December 2017 Available in Internet 23 December 2017 